Conclusion Many colonoscopists appear willing to refer cases to a colleague for EMR, even if it involves transfer to another hospital. Evidence emerged for a small group of experts capable of handling very large polyps, yet referral for surgery remains common. A national referral network might reduce the rate of surgical intervention but while so many colonoscopists perceive themselves to be performing at the "cutting edge" support for this is likely to remain limited.

Disclosure of Interest P. O'Toole: None Declared, J. Anderson: None Declared, J. Geraghty Grant/Research Support from: COOK MEDICAL, R. Valori: None Declared, S. Sarkar: None Declared

\section{PTH-037 DO PATIENTS WITH A PREVIOUS NEGATIVE COLONOSCOPY AND POSITIVE FOB TEST NEED A BOWEL CANCER SCREENING COLONSCOPY?}

doi:10.1136/gutjnl-2013-304907.524

${ }^{1} \mathrm{~K}$ Sahnan, ,."P G Vaughan-Shaw, 'R Valori. 'Department of Endoscopy, Gloucester Royal Hospital, Gloucester; ${ }^{2}$ Department of Colorectal Surgey, Cheltenham General Hospital, Cheltenham, UK

Introduction Patients with a positive faecal occult blood test invited for screening colonoscopy may have undergone previous colonoscopy. Excluding such patients from a repeat colonoscopy may reduce endoscopy waiting lists and avoid repeated and unnecessary invasive investigations. This study investigates the prevalence of previous colonoscopy in Bowel Cancer Screening Programme (BCSP) patients and considers whether repeat colonoscopy is required.

Methods All patients undergoing BCSP colonoscopy over a 30-month period at our unit were identified and cross-referenced against colonoscopy records for the preceding 3 years. New diagnoses of colorectal cancer in the cohort were identified and cancer yield in those with and without recent colonoscopy compared using the chi-squared test.

Results 1419 BCSP colonoscopies were performed in 1339 patients over the study period. 109 colonoscopies were repeats with median interval to repeat 378 days. Indication for prior colonoscopy included prior BCSP invitation $(n=90)$, polyp surveillance $(n=6)$ and symptoms $(n=13)$. There were 111 diagnoses of colorectal cancer in the cohort but no patient with a previous colonoscopy was found to have colorectal cancer. Cancer yield in first time BCSP colonoscopy was greater than in repeated colonoscopy $(8 \%$ vs. $0 \% p=0.002)$.

Conclusion Cancer yield is reduced in BCSP patients with a recent negative colonoscopy. Excluding such patients would reduce pressure on endoscopy units and any morbidity associated with repeat colonoscopy. However, such an approach would be associated with a small risk of missed pathology. Larger studies are required to define the safety of this approach and inform national guidance.

Disclosure of Interest None Declared.

\section{PTH-038 FINDINGS ON BACK-TO-BACK COLONOSCOPIES: AN AUDITABLE STANDARD FOR COLONOSCOPY OUALITY?}

doi:10.1136/gutjnl-2013-304907.525

1."P G Vaughan-Shaw, 'P Rai, 'A Goodman, ${ }^{2} \mathrm{R}$ Valori, 'M Aung. 'Department of Colorectal Surgey, Cheltenham General Hospital, Cheltenham; 'Department of Endoscopy, Gloucester Royal Hospital, Gloucester, UK

Introduction An important marker of colonoscopy quality is detection of pathology and incidence of missed pathology. Back-toback colonoscopies cannot ethically be performed for quality assurance alone yet may be required for clinical reasons. This study aims to investigate the incidence of new findings in colonoscopies repeated within a 12 month period.
Methods All colonoscopies performed over a 3-year period at an Endoscopy training unit were studied. Colonoscopies repeated within a 12-month period were included. Repeats following incomplete colonoscopy were excluded. Data on indication and outcome were collected.

Results 5747 colonoscopies were performed over the study period. 137 repeat colonoscopies were included with median interval 174 days and indications including requirement for endoscopic mucosal resection $(n=47)$, inflammatory bowel $(n=13)$ or polyp surveillance $(n=37)$, previous imaging or endoscopic abnormalities ( $n=15)$, and persistent or new symptoms $(n=25)$. $19(14 \%)$ repeat colonoscopies yielded new findings including 1 new cancer, 234 days following a normal colonoscopy. Additional polyps were identified in 13 colonoscopies indicating a missed polyp rate of $9 \%$. A median number of 2 polyps per colonoscopy with median size $5.5 \mathrm{~mm}$ were found. Crohn's disease $(\mathrm{n}=1)$, and diverticular disease $(n=3)$ were also diagnosed at repeat colonoscopy. There was no morbidity associated with repeat colonoscopy in this series.

Conclusion New pathology was identified in 14\% of repeat colonoscopies. Analysis of clinically indicated repeat colonoscopies and rate of detection of new pathology may offer utility in colonoscopy quality assurance. Larger studies are required to define and validate this criterion as an auditable standard for colonoscopy quality.

Disclosure of Interest None Declared.

\section{PTH-039 SHOULD ANTICOAGULANTS BE STOPPED BEFORE DIAGNOSTIC COLONOSCOPY?}

doi:10.1136/gutjnl-2013-304907.526

1."P G Vaughan-Shaw, 'P Rai, 'A Goodman, 'M Aung. 'Department of Colorectal Surgey, Cheltenham General Hospital, Cheltenham, UK

Introduction With increasing age and polypharmacy, an increasing proportion of patients undergoing diagnostic colonoscopy take anticoagulant medication. Under UK guidelines anticoagulants are continued for diagnostic colonoscopy and which may necessitate a second colonoscopy for 'high risk' procedures after stopping anticoagulants. This may increase endoscopy waiting times and subject often frail patients to a second invasive procedure. This study aims to identify the incidence of and indication for repeat colonoscopy. Methods All colonoscopies performed over a 3 year period were studied. Any patients that underwent 2 colonoscopies within a 12 -month period were included. Data on colonoscopy indication and outcome were collected.

Results 5747 colonoscopies were performed over the study period. Of these, 193 colonoscopies were repeats performed within 12 months. Incomplete colonoscopy $(n=54)$ due to poor bowel preparation $(n=45)$ was the commonest indication for a repeated procedure. Requirement for endoscopic mucosal resection (EMR) or polypectomy indicated a repeat colonoscopy in 48 cases. Patients requiring EMR on warfarin $(n=12)$ or clopidigrel $(n=2)$, accounted for $7 \%$ of all repeated colonoscopies with a median delay repeat colonoscopy of 37 days. There was no morbidity associated with repeated colonoscopy in this series.

Conclusion Repeated colonoscopy due to previous anti-coagulation accounts for a small proportion $(7 \%)$ of repeated procedures and an insignificant proportion $(0.2 \%)$ of all colonoscopies performed. Cessation of anti-colagulation for diagnostic colonoscopy would not result in a significant reduction in endoscopy workload but subject patients to an unnecessary risk of thromboembolic disease.

Disclosure of Interest None Declared. 